Les cénapses lipoprotéiques du lait semblent par ailleurs contenir des protéines solubles (albumine ou globuline-).

Or, les globulines paraissent être le support des anticorps du colostrum.

(A suivre.)

\title{
PRODUCTION ET CONTROLE HYGIÉNIQUE DU LAIT DANS LES ÉTABLES URBAINES ET SUBURBAINES
}

\author{
par \\ ANDRÉ LOUIS \\ Docteur-Vétérinaire sanitaire
}

(Suite)

\section{$\beta$ RECHERCHE DE L'INTÉGRITÉ de LA MAMELLE}

La recherche de l'intégrité de la mamelle est de la plus haute importance, étant donné la fréquence et la gravité de ses altérations. Seflman, de l'Institut de Laiterie de Kiel, estime que sur deux vaches, il n'y en a qu'une dont le pis soit vraiment sain.

$\mathrm{Au} \mathrm{XI}$ e Congrès international de Médecine Vétérinaire, tenu à Londres en 1930 , F. C. Minetr estime que dans certains grands troupeaux, 60 à $80 \%$ des vaches sont atteints de mammites latentes et qu'en général on peut dire que 15 à $40 \%$ des animaux ont leur mamelle infectée par le streptocoque. "

Les altérations de la mamelle présentent une double importance, hygiénique et économique.

Au point de vue hygiénique, les, laits de mammite peuvent être dangereux pour le consommateur.

Au point de vue économique, le préjudice causé est considérable. D'une part, la quantité de lait produite est très fortement diminuée ; d'autre part, la composition chimique de ces laits est telle que leur mélange avec des laits normaux aboutit à des fabrications industrielles de mauvaise qualité, Nous ne citerons qu'un seul chiffre dont l'éloquence se passe de tout eommentaire. Le 9 janvier 1931, devant la Commission plénière du Landtag prussien, le Docteur Steiger, Ministre de l'Agriculture de Prusse déclarait, que pour cet Etat “les dommages imputables à la mammite streptococcique sont évalués, d'après une estimation prudente, à 250 millions de marks-or par an, soit 1.500 millions de franes français».

\section{SES DIFFICULTÉS}

Lorsqu'on a affaire à une affection tapageuse qui se traduit par des signes cliniques évidents, par une modification notable de la 
sécrétion lactée, le diagnostic est facile, et l'intervention du vétérinaire est inutile, car le lait est à ce point modifié qu'il n'est plus marchand. Dans les mammites aiguëes ou subaiguës, le lait est, en effet, remplacé par un liquide jaunâtre ou sanguinolent, grumeleux, de saveur salée ou amère, d'odeur souvent fétide. Les grumeaux, que l'on trouve dans la sécrétion de la mamelle malade, sont formés de matières azotées, d'hématies, de leucocytes et de microbes plus ou moins agglomérés : il ne s'agit plus de lait, mais d'une sécrétion pathologique assimilable aux exsudats et au pus.

Il n'en est pas de même dans les mammites chroniques où la sécrétion a souvent gardé son aspect normal ; le liquide est opalescent comme à l'accoutumée et le goût ne paraît pas modifié ; les symptômes eliniques sont discrets ou inexistants. L'action du vétérinaire acquiert ici toute son importance, ear bien que I'on ait affaire à des mammites discrètes, ce sont les plus dangereuses; nous trouvons, en effet, parmi celles-ci, les mammites tuberculeuses et les mammites streptococciques. Il y a donc intérêt à les dépister dès leur début, avant que des lésions importantes aient pu se constituer, par conséquent, avant que l'examen clinique puisse nous les révéler.

\section{DÉPISTAGE DES MAMMITES PAR L'EXAMEN DU LAIT A LA SORTIE DE LA MAMELLE}

La mise en évidence, au laboratoire, d'un indice de catalase élevé, sur un lait de mélange prélevé à l'étable, permet de suspecter la présence, dans cette exploitation, d'une ou de plusieurs vaches atteintes de mammites. Pourtant, lors de l'examen systématique du cheptel de l'étable suspecte, le lait peut avoir conservé ses caractères physiques normaux, et il est impossible de déceler cliniquement la ou les vaches atteintes.

L'envoi au laboratoire de prélèvements effectués sur tous les animaux d'une étable était irréalisable, S'inspirant des travaux de G. J. Hucker et P. A. Hansen, M. Beaufrìre, alors chef de secteur au contrôle des œufs et du lait, M. GELY, chef de secteur au contrôle de la production laitière et M. THieulin, chef du laboratoire du lait au Service Vétérinaire Sanitaire de la Seine, ont mis au point une méthode permettant de déceler à l'étable, à l'aide d'épreuves simples, les animaux suspects de mammite plus ou moins discrète.

Ces épreuves, restées trop souvent jusqu'ici du domaine du laboratoire, sont: l'essai au bleu de bromothymol et la recherche de la catalase. Elles permettent de mettre en évidence des laits anormaux que leur aspect physique ne trahit pas. Ces anomalies ne sont d'ailleurs pas toujours pathologiques, aussi un examen de laboratoire doit-il intervenir pour donner les précisions nécessaires. 
Dès qu'il en eût les moyens, le Service Vétérinaire de la Seine (directeur M. Ṕ́RARD), utilisant ces deux épreuves, entreprit la recherche systématique des mammites dans les étables du département. Au $1^{\text {er }}$ octobre 1944, ses examens avaient porté sur 6.833 vaches laitières. 2.707 prélèvements effectués sưr 1.457 vaches fournissant un lait anormal, avaient fait l'objet d'examens cytologiques et bactériologiques. L'expérience est d'envergure. Ces méthodes ayant fait leurs preuves et donné des résultats plus qu'encourageants, le temps de la période d'essai est passé. Il importe d'en généraliser l'emploi, en commençant par nos étables urbaines et suburbaines. Elles constitueront autant de "stations d'essai " pour la documentation de nos praticiens.

\section{a). L'examen à l'étable}

Cet examen comporte :

La mise en évidence d'une modification du $p \mathrm{H}$ par l'essai au bleu de bromothymol;

La mise en évidence d'un indice élevé de catalase ;

L'examen des caractères physiques du lait;

L'examen clinique de la mamelle.

Les examens auront lieu sur place, à l'étable, dès les premières heures de la matinée. La traite du matin venant d'être effectuée, la vacuité de la mamelle en facilite l'exploration clinique.

Pour chaque animal, l'examen sera effectué quartier par quartier, car, l'unité sur laquelle doivent porter nos investigations n'est plus la mamelle entière, mais le quartier. Quelques centimètres cubes de lait, recueillis dans un tube à essai, suffiront aux examens.

Personnel et matériel nécessaires.

La mise en œuvre des techniques utilisées à l'étable nécessite pour opérer avec rapidité le concours de deux aides. L'un surveille les prélèvements de lait, trayon par trayon, et apporte les tubes au vétérinaire qui fait les réactions. Le deuxième aide rince les tubes de prélèvements à l'eau ordinaire puis à l'eau distillée ; il lave et essuie les lames.

Le matériel pour l'examen de 40 à 50 vaches peut être contenu dans une petite mallette. Il comporte :

Des tubes à essai stériles rangés dans un tambour.

Les tubes stériles destinés à contenir le lait anormal qui doit être soumis à l'examen du laboratoire, seront munis d'une étiquette sur laquelle on inscrira :

10 Le nom du propriétaire de l'étable;

$2^{\circ}$ Le numéro d'ordre de l'animal dans l'étable; 
$3^{\circ}$ Le quartier d'où provient le lait contenu dans le tube.

Deux porte-tubes métalliques qui servent, l'un d'égouttoir, l'autre pour transporter les prélèvements.

Deux boîtes de cent lames de verre porte-objet.

Un flacon compte-goutte de solution orange de bleu de bromothymol (solution à $5 \%$ dans l'alcool à $60^{\circ}$ ).

Un flacon contenant une réserve de solution alcoolique de bleu de bromothymol.

Un flacon eompte-goutte d'eau oxygénée à 4 volumes (solution d'eau oxygénée officinale diluée à $40 \%$ ).

Un flacon contenant une réserve d'eau oxygénée à 4 volumes.

Un ou deux flacons d'eau distillée.

Une boîte d'étiquettes gommées.

\section{MISE EN ÉVIDENCE D'UNE MODIFIOATION DU $p H$ PAR L'ESSAI AU BLEU DE BROMOTHYMOL}

L'acidité ionique du lait normal à la sortie de la mamelle est à peu près fixe ( $p \mathrm{H}=6,6$ environ). Si elle est nettement augmentée $(p \mathrm{H}=6,8$ ou 7$)$ ou diminuée $(p \mathrm{H}=6,4$ ou 6,2$)$, elle révèle un trouble grave.

La composition chimique du lait de mammite est différente de eelle du lait normal. L'acidité est en principe diminuée et l'on peut poser en règle générale que toute mamelle malade donne une sécrétion plus alcaline. Cependant, le phénomène peut être masqué, notamment dans la mammite streptococcique, par la fermentation lactique que peut provoquer Streptococcus mastitidis. On aura dans ce cas un lait dont l'acidité sera soit normale, soit même augmentée. Par contre, dans la mammite tuberculeuse où la fermentation lactique ne masque pas l'alcalinisation de la sécrétion, on constate toujours une acidité diminuée.

En résumé, dans la majorité des cas, les laits de mammite présenteront une acidité ionique anormale, et leur simple examen de ce point de vue, par l'essai au bleu de bromothymol, permettra de les dépister.

L'épreuve au bleu de bromothymol doit être effectuée sur du lait frais, n'ayant pas subi un début de fermentation lactique. C'est pourquoi on opère à l'étable et non au laboratoire.

Principe. - Le bleu de bromothymol présente une zone de virage pour des valeurs du $p H$ voisines de celles du lait frais normal, soit $p \mathrm{H}=6,6$ environ, vers $15^{\circ}$ centigrade.

L'emploi d'un tel indicateur permet de se rendre compte immédiatement si un lait frais a un $p H$ normal, ou trop faible (laits acides), ou trop élevé (laits alcalins). 
Matériel. - Lames de verre porte-objet; solution orange de bleu de bromothymol à $5 \%$ o dans l'alcool à $60^{\circ}$ (en flacon comptegouttes).

Technique. - L'opérateur dispose, sur une table ou un support horizontal de couleur neutre, 8 lames de verre, deux pour chaque quartier. Le lait, récolté toujours dans le même ordre (par exemple, quartiers $\mathrm{AD}, \mathrm{AG}, \mathrm{PD}, \mathrm{PG}$ ) est présenté par un aide, en quatre tubes à essai. Tenant les tubes de la main gauche, on dépose une goutte de la solution de bleu de bromothymol, sur les deuxième, quatrième, sixième et huitième lames, puis on fait couler directement sur la goutte de réactif de la lame no 2 une ou deux gouttes de lait du premier tube ; il est procédé de même avec les trois autres tubes sur les lames correspondantes.

Le mélange "réactif-lait » se fait spontanément et le virage est instantané.

Les laits normaux $(p H=6,6)$ donnent une coloration jaune verdâtre pâle ;

Les laits anormaux dont le $p H$ est augmenté $(p H>7$ - laits hypo-acides ou "alcalins ") provoquent un virage au vert ou au vert bleu;

Les laits dont le $p \mathrm{H}$ est diminué ( $p \mathrm{H}<6,2$ - laits plus acides) donnent au contraire une coloration jaune serin.

La simultanéité des épreuves sur les quatre échantillons d'une mamelle facilite la lecture des résultats.

Nous insistons sur le fait que le virage est instantané. Il importe de savoir en effet, que lorsqu'on opère à l'intérieur de l'étable, par suite du dégagement plus ou moins abondant des vapeurs d'ammoniaque provenent des litières, le virage au vert se produit, mais il est lent, progressif, et demande quelques minutes.

Interprétation. - Tout lait présentant un $p H$ "acide " ou "alcalin " est un lait " anormal ».

Sans avoir dans tous les cas une valeur rigoureuse, cette épreuve doit faire penser :

Si le lait est alcalin, à l'existence d'une mammite généralement chronique (tuberculeuse ou streptococcique) ou à une affection aiguë pouvant être banale. Si le lait est acide, à l'existence d'une mammite streptococcique.

Cette méthode, très sensible, sera en défaut chaque fois que la fermentation lactique aura exactement compensé l'alcalinisation pathologique. Aussi pour obvier à cette défaillance aura-t-on conjointement recours à l'épreuve de la catalase sur lame. 


\section{MISE EN ÉVIDENCE D'UN INDIOE ÉLEVÉ DE CATALASE}

L'épreuve de la catalase fut imaginée par TrommsdorfF pour déceler le lait provenant de mamelles anormales.

Principe. - La catalase est une diastase, trouvée dans tous les laits, capable de décomposer l'eau oxygénée, avec libération d'oxygène moléculaire se dégageant à l'état gazeux. Ce dégagement est d'autant plus rapide que la concentration en catalase est plus élevée et la mesure de son intensité est une précieuse donnée utilisée couramment dans la pratique.

La catalase est, en général, plus abondante dans les laits malades, dans ceux qui ont été reeueillis et manipulés sans précaution, et dans les laits acides et de richesse microbienne élevée. Au contraire, le lait provenant de vaches saines; normalement traité et frais, possède de faibles propriétés catalytiques.

La catalase du lait a une double origine : elle provient d'une part des leucocytes du lait (polynucléaires), d'autre part des microbes et ferments du lait.

On éliminera l'action de la catalase microbienne en opérant sur du lait aussi frais que possible. En opérant à l'étable on ne mettra en évidence que la catalase leueocytaire.

On appelle indice de catalase le nombre de centimètres cubes d'oxygène (mesurés à la température et à la pression ambiante) libérés en deux heures par $100 \mathrm{~cm}^{3}$ de lait, en présence d'eau oxygénée (le mélange de lait et d'eau oxygénée étant maintenu à $30^{\circ}$ centigrade).

Pour le lait frais normal (d'acidité inférieure ou égale à $20^{\circ}$ Dornic) l'indice de catalase est presque toujours inférieur à 25 et ne dépasse jamais 30.

Si l'indice de catalase dépasse 30 , le lait est suspect.

Matériel. - Lames de verre porte-objet, solution d'eau oxygénée à quatre volumes (en flacon compte-gouttes).

Technique. - Cette épreuve est effectuée en même temps que la précédente. Quelques gouttes de lait du premier tube ont été déposées sur la lame $n^{\circ} 1$, en même temps qu'on opérait le mélange de ce lait avec la solution de bleu de bromothymol sur la lame n० 2 . La même opération a été effectuée avec les trois autres échantillons, dans les mêmes conditions. Les lames no $1,3,5,7$, présentent une plage lactée au centre de laquelle on verse une goutte d'eau oxygénée à quatre volumes.

Par temps froid, le flacon compte-gouttes contenant l'eau oxygénée doit être maintenu à une température modérée ; on le conservera, par exemple, dans une poche de gilet, jusqu'au moment 
même de l'emploi - le froid gênant ou retądant la réaction et pouvant fausser le résultat de l'épreuve.

La réaction diastasique demande deux à quatre minutes pour s'effectuer ; au bout de ce temps, les lames de lait dont la catalase est anormalement abondante, présentent de nombreuses et fines bulles d'oxygène très visibles à la lumière du jour. Si l'étable est obseure, il est recommandé d'éclairer avec une lampe électrique de poche, les lames ayant été placées sur fond neutre.

Interprétation. - Tout lait présentant ce dégagement gazeux est un lait dont l'indice de catalase est certainement supérieur à 30 et on se trouvera en présence soit de colostrum, soit de sang, soit enfin de leucocytes polynucléaires très abondants et traduisant une présomption de mammite.

Il s'agit dans ces trois hypothèses d'un "lait anormal".

Cette épreuve nous permet donc de déceler les "laits anormaux " qui auraient pu échapper, par suite d'une acidification compensatrice d'origine streptococcique, au crible de l'essai au bleu de bromothymol.

On pourrait être tenté, pour confirmer le diagnostic de "lait anormal ", de n'effectuer la catalase sur lame que sur les laits à $p$ H modifié. Ce serait une erreur. S'il n'est pas possible de pratiquer cette épreuve sur tous les laits, on la fera obligatoirement sur ceux dont le virage au bleu de bromothymol est peu marqué (cette épreuve lèvera tous nos doutes) et sur tous ceux qui présentent un $p \mathrm{H}$ normal. On pourra, à la rigueur, la supprimer sur les laits dont le $p H$ est modifié et qui de ce fait sont déjà classés anormaux.

Prélèvement. - Tout lait présentant un $p \mathrm{H}$ acide ou alcalin ou un indice élevé de catalase est un "lait anormal». Il fera l'objet d'un prélèvement aseptique,

On n'éliminera pourtant pas, comme il est prescrit, quelques jets de lait avant d'effectuer le prélèvement, car, très souvent, lorsque la traite vient d'avoir liev, on aura des difficultés à se procurer la petite quantité de lait nécessaire au laboratoire (un tube à essai, soit $25 \mathrm{~cm}^{3}$ environ). Ce prélèvement sera néanmoins aseptique, car la traite qui vient d'être effectuée et les quelques jets de lait pris pour l'examen à l'étable remplissent le même but, à la condition d'opérer le prélèvement dès que le lait est reconnu anormal. En aucun cas, il ne faut attendre d'avoir éprouvé tout le cheptel de l'étable pour effectuer, en fin de séance, les prélèvements des laits anormaux, car, entre temps, les animaux ont pu se coucher et souiller leurs trayons. 


\section{EXAMEN DES CARACTERES PHYSIQUES DU LAIT}

L'examen des caractères physiques doit être fait en même temps que sont réalisées les deux épreuves précédentes.

Dans le tube à essai, sont mis en évidence les laits "grumeleux ", les laits "hémorragiques", les laits dont la coloration et d'une façon générale, l'aspect sont modifiés.

L'étalement en couche mince, sur lame (préparation à l'épreuve de la calatase), permet à lui seul de déceler une eatégorie de laits qui échappent à l'observation du trayeur et dont les caractères n'apparaissent pas dans le tube à essai immédiatement après la traite : ce sont les laits "clairs", "grisâtres ", "séreux" pouvant avoir l'aspect de laits écrémés et mouillés. Conservés dans le tube de prélèvement, ils permettent souvent d'observer, après un temps variant de dix à soixante minutes, une sédimentation spontanée le lait se séparant en trois couches ; en surface la crème, au fond un dépôt plus ou moins abondant de particules ténues( matières protéiques précipitées), entre les deux, le lacto sérum clair.

Tout lait dont les caractères physiques sont modifiés est un lait "anormal ».

\section{EXAMEN CLINIQUe DE LA MAMELLE}

Toute anomalie du lait entraine l'examen clinique de la mamelle, cet examen pouvant d'ailleurs être systématiquement pratiqué dans tous les cas. Il doit avoir lieu immédiatement après la traite, car il n'est pas satisfaisant quand la mamelle est distendue par le lait.

Les commémoratifs fournis par le trayeur seront précieux. Toute vache dont la production laitière a diminuée assez rapidement, sans que rien du côté alimentaire ne permette d'en donner l'explication, sans que son état général soit modifié, sera suspecte. Lorsque, sans raison apparente, la séçrétion des quatre quartiers est affectée dans des proportions semblables, il faut penser à une infection générale. Au contraire, quand ce n'est que la production isolée d'un quartier qui est atteinte, il faut penser à une mammite qui apparaît, à une infection latente qui se réveille.

Lors de l'exploration de la mamelle, nous n'oublierons pas que ce sont les quartiers postérieurs, particulièrement celui de gauche, qui sont le plus souvent atteints.

SVEN WALL qui a étudié la tuberculose mammaire, montre que l'infection de la moitié gauche de la mamelle est plus fréquente que celle de la moitié droite et que les quartiers postérieurs sont plus souvent atteints que les quartiers antérieurs. Sur 115 mamelles qu'il a examinées, quartier par quartier, dans 105 cas, soit $91 \%$, la tuberculose a frappé plus particulièrement un ou deux quartiers postérieurs. 
Un travail très intéressant de Nоттвонм montre que dans la mammite ce sont les quartiers postérieurs qui sont le plus souvent envahis, Ils le sont dans $83,33 \%$ des cas. Examinant ensuite s'il y a une différence entre le quartier postérieur droit et le quartier postérieur gauche, il constate que sur ces $83,33 \%, 61,11 \%$ appartiennent au quartier gauche et $22,22 \%$ au quartier droit. Il explique cette différence de la façon suivante : "Le fait que les trayons postérieurs sont plus souvent infectés que les autres, tient à ce que, plus que les antérieurs, ils stagnent dans les matières excrémentitielles et aussi à ce que, au repos, ils se trouvent comprimés davantage que les trayons antérieurs entre les cuisses.

Pourquoi le quartier gauche est-il plus souvent atteint que le droit ? Cela serait dû à ce que la plupart des bovins choisissent plutôt le côté gauche que le droit pour se reposer, en raison de la situation de la panse à gauche. L'animal évite de se coucher sur le côté droit afin de ne pas comprimer le reste des organes digestifs aveo la panse, dont le poids est considérable.

Aux raisons que donne Notrboнm, Porcher en ajoute une autre :

"Nous sommes tentés de penser, dit-il, que la vidange de la glande est moins complète sur le quartier postérieur, plus volumineux, et dont la traite, par suite, est plus fatigante, que sur le quartier antérieur. Les quartiers postérieurs sont donc toujours et plus ou moins en état de rétention; or la rétention est la circonstance favorisante principale de l'infection mammaire, "

En observant la symétrie de la mamelle, la dimension, le poids, la consistance de chaque quartier, et en les comparant avec les autres quartiers, on pourra en noter l'hypertrophie ou l'atrophie : Dans la tuberculose mammaire, il y a toujours hypertrophie de la mamelle; dans la mammite chronique, la mamelle s'atrophie, se ratatine, se sclérose.

A la palpation on pourra noter des modifications du parenchyme (indurations en masse ou en noyaux, sensation de paquets de cordes).

Dans la mammite chronique, l'induration peut-être partielle, nodulaire ou diffuse.

Dans la mammite contagieuse qui est une mammite à évolution subaiguë ou chronique, il se produit des noyaux d'induration, de la grosseur d'une noisette qui font dire aux nourrisseurs que la mamelle est "nouée". Dans la mammite tuberculeuse, la mamelle paraît dure, bosselée, de consistance pierreuse, insensible. Les ganglions rétromammaires peuvent à la palpation offrir les mêmes sensations alors que la mamelle est encore intacte. 
Ces divers signes peuvent entraîner une conclusion clinique ou une suspicion que devra confirmer ou infirmer le laboratoire.

Le plus souvent, le résultat de cette exploration peut paraitre décevant; il n'y a pas de modification de volume de la mamelle dont le tissu glandulaire reste souple et élastique sans présenter les moindres indurations limitées ou diffuses. L'examen eliniques se révèle impuissant à déceler la mammite que les épreuves au bleu de bromothymol et la catalase font suspecter et que l'examen de laboratoire confirmera. Il n'y a pas lieu de s'en étonner. G. et R. Moussu traitant de la mammite streptococcique disent en effet : "La première manifestation appréciable se traduit par une modification dans la sécrétion lactée. Le lait diminue de quantité, il ne semble pas ou peu altéré à première vue, mais si on le laisse reposer, il se coagule rapidement. "Nous savons, en effet, que Streptococcus mastitidis peut provoquer une fermentation lactique qui augmente l'acidité du lait. "L'infection, poursuit Moussu, existe déjà sans que rien ne la traduise à l'examen de la mamelle. Le symptôme local qui apparaît ensuite est la présence d'un nœud ou noyau d'induration scléreux situé au-dessus du trayon dans l'épaisseur de la mamelle. Ce nœud, de forme arrondie ou ovoïde, mal délimité à la périphérie, augmente progressivement sans signe d'inflammation aiguë. Le processus scléreux ne marche qu'avec une extrême lenteur ; au bout de plusieurs mois la sclérose ne porte souvent que sur un tiers de la hauteur des quartiers envahis."

Si done l'examen clinique a paru souvent décevant, e'est que nous avons en mains des tests d'une grande sensibilité qui permettent de déceler les mammites tout à leur début, alors que les lésions et par conséquent les symptômes n'ont pas eu le temps de se constituer. Cette méthode est si sensible, qu'elle nous révèle non seulement les mammites tout à leur début, mais encore les "prémammites», si l'on peut dire, et parmi elles, les "mammites latentes " de Porcher. Elle nous donne l'alarme, dès qu'un trouble physiologique se produit dans la mamelle, trouble dont la persistance et l'aggravation provoqueraient la mammite. On n'ignore pas le rôle joué par la "rétention " qui "fait le lit de la mammite "

On ne sera done pas surpris des "soi-disant défaillances" de cet examen clinique.

\section{b) L'examen au laboratoire (1)}

Tout lait reconnu suspect (caractères physiques apparemment modifiés, épreuve de la catalase positive, $p \mathrm{H}$ alcalin ou acide) donne

(1) Pour plus amples détails sur les différentes techniques, consulter ; G. THreuLrd et R. Vuinlaume : "Eléments pratiques d'analyse et d'inspection du lait ». 1 vol. de 206 pages, $15 \times 25$. Le Lait, éditeur, 16, rue Spontini (XVI), Paris, 1942. 
immédiatement lieu à un prélèvement aseptique qui sera apporté au laboratoire dans les plus brefs délais, chaque tube ou flacon portant indication d'origine. Ces prélèvements sont- accompagnés d'une fiche numérotée portant le signalement de l'animal et les résultats de l'examen à l'étable (caractères physiques du lait, $p \mathrm{H}$, catalase, et constatations cliniques).

Chaque prélèvement recueilli aseptiquement est centrifugé ; à partir du culot de centrifugation, il est procédé à un ensemencement en bouillon de viande lactosé et à la préparation de deux frottis dont l'un sera coloré par la méthode de Gram et l'autre au bleu de méthylène.

Le frottis coloré au bleu de méthylène permet l'appréciation de la formule leucocytaire (le rapport $\frac{\text { mononucléaires }}{\text { polynucléaires }}$ voisin de un et toujours supérieur à 0,5 dans un lait normal) et la recherche des éléments anormaux (gros mononucléaires lipophages, corps granuleux, corps en croissant). L'abondance des polynucléaires altérés (pus) révèle l'infection; la présence des éléments anormaux (gros mononucléaires lipophages au protoplasma bourré de globules gras, corps granuleux) témoigne de l'état de rétention (trouble encore physiologique, mais favorable à l'infection dont il constitue souvent la première étape).

Le frottis coloré par la méthode de Gram montrera éventuellement la présence de Streptococcus mastitidis de Nocard et Mollereau, agent de la mammite contagieuse. Il se présente sous la forme de streptocoques à longues chaînes (plus de 15 éléments, normalement 50 à 100), gardant irrégulièrement le Gram, souvent enchevêtrés en formant de véritables pelotes. On notera, en même temps, la présence de polynucléaires altérés ou non ; leur abondance est un signe évident d'infection mammaire.

Cultures. - Après vingt-quatre heures de séjour à l'étuve à $37^{\circ}$ centigrade, les tubes de bouillon sont examinés. Généralement, Streptococcus mastitidis forme des flocons (culture en mie de pain) sans troubler le milieu et donne rapidement après seize à vingt quatre heures, un sédiment avec liquide clair surnageant, tandis que les germes saprophytes de la mamelle troublent le bouillon ; mais, ces caractères n'ont qu'une valeur de présomption, les cultures étant très rarement pures.

A l'aide d'une pipette Pasteur, on opère un prélèvement au fond de chaque tube de culture (de façon à intéresser le dépôt formé) et portant quelques gouttes sur une lame porte-objet, on effectue, après dessiccation et fixation, une coloration de Gram. L'examen microscopique consécutif permettra de noter, avec les cocci sapro- 
phytes (mammocoques, entérocoques...) la présence éventuelle de longues chaînettes (plus de 15 éléments et normalement 50 à 100) gardant irrégulièrement le Gram, formées par Streptococcus mastitidis.

La coexistence de ces longues chaînettes dans la culture et de polynucléaires abondants dans les frottis obtenus à partir du culot de centrifugation permet de porter le diagnostic de mammite streptococcique.

Ce test, basé sur la morphologie des éléments microbiens, n'est pas absolu car certaines souches de Streptococcus mastitidis ne forment que des chaînettes très courtes ou des diplocoques, et, dans les conditions présentes, passeront inapercues.

Pratiquement, les frottis obtenus à partir des culots de centrifugation décèlent $20 \%$ des cas de mammite streptococcique tandis que les cultures permettent le diagnostic de $80 \%$ d'entre eux environ. Si l'on veut bien considérer que le résultat est acquis en vingt-quatre à quarante-huit heures, on reconnaîtra à cette technique un réel intérêt, ear la recherche des $20 \%$ des cas qui nous échappent exige une technique longue et délicate qui sort du domaine de la pratique courante.

\section{c) Suites à donner à ces examens CATEGORISATION DES ANIMAUX}

Dès le premier triage, réalisé lors de l'examen à l'étable, il est constitué un premier groupe (groupe A) d'animaux reconnus " apparemment sains " et déclarés tels puisque ne présentant aucun quartier suspect.

Après l'examen de laboratoire, les autres animaux de l'étable peuvent être répartis en trois groupes : un groupe (groupe B) renferme les animaux suspects, fournissant un lait anormal, dû soit à un trouble physiologique (rétention) ou à un léger trouble pathologique non spécifique (mammites latentes de Porcher). "

Les deux derniers groupes rassemblent les sujets déclarés atteints de mammite.

Ce sont : le groupe Créunissant les animaux atteints de mammite banale (lait aux caractères physiques apparemment modifiés ou non, avec du pus décelé au microscope) et le groupe D renfermant tous les sujets atteints de mammite streptococcique.

On pourra maintenir dans l'étable, en les séparant, les trois premiers groupes, mais les animaux du groupe D, atteints de mammite streptococcique, devront être isolés, par suite de leur contagiosité, dans le "local d'isolement ». 


\section{UTILISATION DU LAIT}

Le lait des vaches du groupe A composé d'animaux sains est libre, sans condition.

Le lait des vaches du groupe B renfermant les animaux suspects, à lait anormal, ne peut être vendu, pour la consommation humaine, qu'après pasteurisation ou ébullition prolongée pendant cinq minutes.

Le lait des vaches des groupes C et D ne contenant que des animaux malades, atteints soit de mammite banale soit de mammite streptococcique, ne pourra être livré qu'à la consommation animale, à la ferme même où il est produit, et seulement après chauffage (cuisson comprenant une ébullition prolongée pendant au moins cinq minutes).

Les quartiers d'une même mamelle étant microbiologiquement indépendants l'un de l'autre (W. STECK) et chaque quartier représentant une glande distincte, nous pourrions, logiquement, envisager l'utilisation du lait, non plus en fonction de l'animal, mais en fonction du quartier. C'est ce que font GéLY et THIEuLIN qui en permettent l'utilisation de la façon suivante :

Lait de vente libre. - Le lait des animaux sains et celui provenant des quartiers sains des animaux suspects ou atteints de mammite banale.

Lait obligatoirement chauffé. - Le lait des quartiers suspects du groupe $B$, celui des quartiers atteints de mammite banale (à condition que les caractères physiques ne soient pas apparemment modifiés), celui des quartiers non atteints des animaux à mammite streptococcique.

Lait ne pouvant être livré (et seulement afrès chauffage) que pour la consommation animale à la ferme où il est produit. - Les laitsaux caractères physiques apparemment modifiés et ceux des quartiers atteints de mammite streptococcique.

Cette façon d'envisager l'utilisation du lait est plus théorique que pratique. Son application, lorsque le nombre des animaux malades ou suspects est tant soit peu élevé, devient pour le trayeur un véritable casse-tête chinois, alors qu'il est si simple pour le vacher de se rappeler simplement que tout le lait des animaux parqués dans cette partie de l'étable peut être consommé librement, que celui des vaches de ce coin-ci ne peut être consommé qu'après chauffage, et que celui des vaches de ce coin là ne peut être utilisé pour l'alimentation humaine.

Il s'agit là de mesures applicables en temps de disette. Ce sont, disent leurs auteurs, des mesures "minima» limitées par les cir- 
constances actuelles où il importe d'utiliser pour l'alimentation humaine tout ce qui peut être récupéré.

Il convient de les proserire en période normale.

\section{DiRECTIVES POUR LA PROPHYLAXIE ET LE TRAITEMENT DES MAMMITES}

Nous ne saurions trop insister sur le caractère infectieux de la mammite streptococcique. "Une vache atteinte peut être considérée comme dangereuse pour les autres et être isolée. On la traira la dernière et on anéantira sa production (G. Collumbien, $10^{\mathrm{e}}$ Rapport annuel de la Frise, Pays-Bas). "

"Cet isolement complet et absolu des malades est, au point de vue prophylactique, le moyen le plus sûr et le plus efficace, très supérieur à celui qui recommandait autrefois de pratiquer la traite sur les animaux malades en dernier lieu (Moussu). ”

Le local d'isolement que nous avons prévu comme annexe de notre vacherie nous sera, dans ce cas, d'une utilité incontestable.

Les emplacements précédemment occupés par les animaux malades devront être désinfectés.

Nous n'émettrons pas d'opinion sur les différentes thérapeutiques des mammites, plus ou moins incertaines, et nous ferons appel à la méthode préventive. La protection des mamelles saines est plus importante que le traitement proprement dit des mammites et un soin tout particulier doit être donné aux mamelles produisant un lait anormal, encore physiologique, afin qu'elles puissent recouvrer leur intégrité.

La mammite, selon une expression pittoresque recueillie par Gély et THieunin, est une véritable "maladie du poignet ". Les traites complètes, régulièrement espacées, sont le facteur fondamental de l'entretien de la sécrétion lactée et de la santé de la mamelle.

Une traite incomplète laisse, dans le sinus, dans les gros canaux galactophores et même et surtout dans les plus fins, e'est-à-dire jusque dans la profondeur de la glande, un aliment extrêmement favorable à la culture tant du streptocoque que des microbes, cocci principalement, qui sont habitués à vivre au sein de la mamelle, mais qu'une traite régulièrement effectuée, complète chaque fois et faite à temps, empêche de cultiver.

Puisque la rétention favorise l'infection, la contre-partie, c'està-dire la multiplicité des traites, devra conduire ou du moins aider à la guérison en détergeant la glande, en la vidant chaque fois de son contenu aussi complètement que possible, en enlevant par conséquent à la flore existante le milieu de culture qu'elle préfère, par le balayage vigoureux des voies de sortie du lait. 
La traite fréquente et à fond réduira nos groupes B (lait anormal) et $\mathrm{C}$ (mammite banale) et nous permettra de récupérer très rapidement ces animaux.

Chaque quartier doit être sollicité "à fond" pour vider la glande, même si le lait, inutilisable, doit être détruit.

On commencera la traite par les animaux sains, on continuera par les suspects, puis par les mammites banales, pour terminer par les mammites contagieuses, afin d'éviter les contaminations classiques par le trayeur.

Tout animal présumé guéri, sera, avant de reprendre sa place à l'étable, l'objet d'un nouvel examen cytologique et bactériologique.

$\mathrm{Au}$ contraire nous éliminerons, le plus rapidement possible, toutes les vaches atteintes de mammite streptococcique, qui, même après guérison, peuvent constituer de redoutables " porteurs de germes ».

En effet : «Lorsqu'une glande a été infectée par le streptocoque, on peut dire, d'accord avec tous les auteurs, qu'elle le reste, pendant fort longtemps, sinon durant toute la carrière économique de l'animal. Le quartier reste indéfiniment, peut-on dire, le siège d'une culture très ralentie de streptocoques. Ceux-ci, cantonnés dans la glande après que l'infection visible a disparu, peuvent être le point de départ d'une nouvelle poussée et les microbes, dès lors plus virulents, éliminés avec la traite pourront ainsi servir à réinfecter des animaux voisins, indemnes jusque là (PoRchír). »

\section{Conclusion}

En parfait accord avec THIEULIN (1) nous ne tolérerons pour la vente à l'état cru "que le lait provenant de vaches en parfait état sanitaire et notamment indemnes de tuberculose, de mammite, d'affections aiguës ou chroniques des voies génitales ou intestinales ".

(A suvve)

(1) A. Plenndoux. "Manuel de législation sanitaire "vétérinaire" ; "Projet d'arrêté municipal concernant l'hygiène du lait et son contrôle s, pary. 